\title{
KINEMATICS AND DYNAMICS OF OH/IR STARS
}

\author{
H. DEJONGHE \\ Sterrenkundig Observatorium, Universiteit Gent, Belgium
}

\begin{abstract}
The available kinematical data on $\mathrm{OH} / \mathrm{IR}$ stars is discussed, and dynamical models for the $\mathrm{OH} / \mathrm{IR}$ stars are presented.
\end{abstract}

\section{Introduction}

In this contribution I would like to present you one particular way of modelling the kinematics of a tracer population of stars in our Galaxy. This contribution is a continuation of the previous one. One of the things that I hope to convince you of is that the definition of such a thing as "a dynamical model for the OH/IR stars", involves quite a number of elements. Therefore I will highlight in boldface every item that is essential in defining the models presented here.

Circular orbit models will not work as representations of the kinematics of $\mathrm{OH} / \mathrm{IR}$ stars, and this is immediately obvious from Figure 1. There you see a familiar $(l, v)$ plot for a sample of OH/IR stars, compiled by Peter te Lintel Hekkert, who, very unfortunately and unexpectedly, was unable to attend this symposium in order to present these results himself. I will call it the tLH sample. It consists of almost 900 stars.

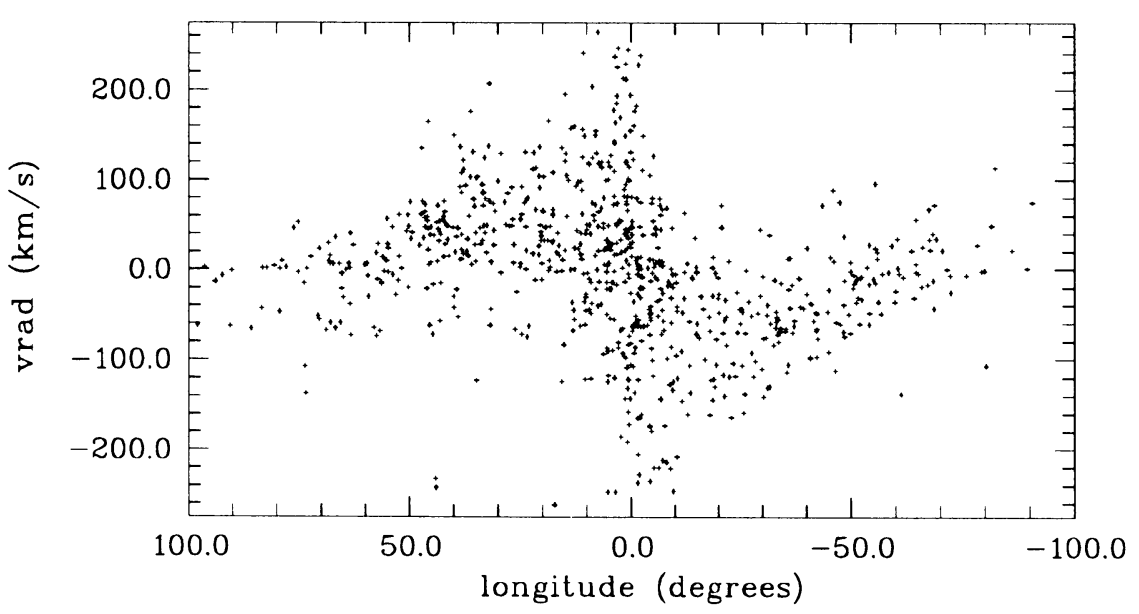

Fig. 1. The $(l, v)$ diagram of the L sample. Note the counterrotators.

The smoking gun in Figure 1 is the presence of counterrotating stars (especially in the Bulge region), which, in any case, point to orbits that are not simply circular. It is nice to see this come out straight from the data, though nobody will argue with the statement that we cannot expect the stars in the Bulge to form only a thin disk on these small scales. By the way, I'll tentatively define the Bulge here as 
a "not further specified volume inside a $3 \mathrm{kpc}$ radius"... In any case, the greater variety in the orbits there does not mean that a thin disk with stars in circular orbits isn't there (after all, our solar system is a great example of a thin disk on even smaller scales), but I'll return to this in a while.

I will not expand on the way the data in Figure 1 were acquired, nor on the physics of the OH/IR's that we can learn from it, since these topics are covered by Habing (this symposium) and can also be found in te Lintel Hekkert (1990,1991a). Suffice it to say that this particular data set is a compilation from catalogues by Eder et al. (1988), te Lintel Hekkert et al. (1991a) and Sivagnanam et al. (1989), all based on the IRAS PSC from which positions were selected according to infrared colours, fluxes $(f)$ and IRAS flux qualities. Essentially, you see here all sources with colours R21 $=f_{25 \mu \mathrm{m}} / f_{12 \mu \mathrm{m}}$ between 0 . and .9 . This was done in an attempt to obtain a homogeneous sample of $\mathrm{OH} / \mathrm{IR}$ stars with thick dust shells and a reasonably constant bolometric correction $f_{t o t} / \nu / f_{12 \mu m}$. Additional selection criteria were the presence of the two regular $1612 \mathrm{MHz}$ peaks which had to be separated by more than $10 \mathrm{~km} / \mathrm{s}$, and the requirement that the $f_{12 \mu m}$ had to be larger than $3 \mathrm{Jy}$. Finally, one must keep in mind that samples based on IRAS data are incomplete in the areas where IRAS was confusion limited. The models therefore will not include data from a region with approximate boundaries $|l|<45^{\circ}$ and $|b|<2^{\circ}$.

Simple models are great to answer simple questions, and such questions are likely to be of particular interest to the physicist inside the astronomer: determination of the rotation curve, estimates of the total mass, the total power emitted, etc... And yet, even the progress on these questions is slow. This is due partly because any adequate theory tends to become complex nevertheless, partly because some parameters are hard or impossible to measure (such as stellar ages, distances), partly because of the nagging problem of the extinction, partly because the parameters of interest are often statistical properties of samples (e.g. determination of the local standard of rest), and finally because there are so many interrelated parameters. It would seem that one really needs a "model for everything". Such may be a great proposal for ambitious nationals of wealthy countries who aspire large grants from well-grown funding agencies, but it's simply not very realistic.

All the problems mentioned above are present in this contribution. I will not address them any further, since this would be restating the obvious. But allow me to expand on something else: why, apart from the above question, could one be interested in dynamical models in the first place? I think that, for the cartographer inside the astronomer, it's a natural thing to do. In order to motivate this opinion meaningfully, we must agree on what we mean by dynamical modelling.

\section{Elements of Dynamical Modelling}

The equations that govern the motion of $10^{11}$ starlike objects in mutual interactions and the interpretation of their solutions are hopeless. Both theory and assumptions will be needed to make them suitable for human grasp. It is a well-known textbook topic (e.g. Binney \& Tremaine 1987) that in most cases of astrophysical interest it suffices to simplify the motion of each of these objects to the motion of one (hypothetical) object, moving in the gravitational field which is generated collectively by 
all the others. This reduces phase space from $6 \times 10^{11}$ coordinates to 6 coordinates, together with a potential function that is loosely coupled by an integral operator to the contents of that space, which is characterized by a distribution function, which is a probability density in phase space.

The potential generates structure in phase space, because it creates orbits. The quintessential orbit is the linear harmonic oscillator, e.g. a spring. At every moment it has a length $z$ which changes at a rate $v_{z}$. Phase space is the 2-dimensional space $\left(z, v_{z}\right)$. If we do not know the dynamical state of the spring completely, it is natural to ask what information we can single out as particularly important. The maximum length $z_{m}$ must be such a quantity, because then, at least, we can confine the length of the spring, though we've lost the ability to predict its actual length at any particular moment. This $z_{m}$ is an example of an integral of the motion: it is a function of phase space coordinates that remains a constant along the orbit, and therefore it can be used as a label for that orbit. Hence, we can now describe the linear harmonic oscillator with $z_{m}$ (a constant) and only one rapidly changing coordinate ( $z$, or $v_{z}$, or something else).

In 3 dimensions, one would expect 3 constants of the motion and 3 rapidly changing variables for every orbit. This is true for integrable potentials, by definition. Most potentials however are not integrable, but it is likely that for most astrophysical purposes there exist good integrable fits (Goodman and Schwarzschild 1981, Dejonghe \& de Zeeuw 1988), safe possibly for tumbling triaxial figures. A very elegant class of integrable potentials are the Stäckel potentials, which have the nice property that the integrals of the motion are quadratic functions of the velocities.

In order to better understand the significance of these integrals, let's consider the following experiment. We affix many springs to a flat surface. The springs only vibrate in the $z$-direction (perpendicular to the surface), and hence their $x$ and $y$ coordinates which are markers on the surface are constants of the motion. Now we disturb the springs, for example by pushing them down simultaneously by hand. The imprint of the hand will be lost very quickly, and in the analysis of the resulting dynamical state, it will certainly not matter very much to focus on the description of the rapidly changing coordinates. If we only knew $z_{m}(x, y)$, then we would know the profile of the perturber, which is everything that there is to know in this experiment.

Consider next the somewhat different situation that at every location $(x, y)$ there are a lot of springs (for example molecules), which may or may not start to vibrate due to infalling light, then the number of excitations $N$ will be proportional with the intensity, while the degree of excitation (the $z_{m}$ ) will tell us something about the wavelength of the infalling light. The function $N\left(x, y, z_{m}\right)$ we call a distribution function. It is written here as a function in integral space. It cannot exist without a medium for which it is a probability density, though it may provide us with important information on something else (the infalling light). This function is very analogous to the concept with the same name in stellar dynamics; the medium there is called a tracer population. On a photographic plate, the distribution function is a faithful representation of the perturbing radiation, and the resulting picture is its own justification. In stellar dynamics, and now comes a more personal view, the distribution function could serve the same purpose: it is "simply" a picture of the 
stellar component. By analogy with ordinary pictures, this picture may very well be its own justification. One is not necessarily unimaginative if no more questions are asked, just as one does not ordinarily inquire about the "origin and evolution" of some feature in a portrait when looking at it...

Of course, it is hoped that the distribution function will teach us a lot about the origin and evolution of galaxies and bulges.

\section{The input: data and assumptions}

My first assumption is far reaching: I'll assume that the data can be modelled by an equilibrium model. This is to a lesser extend a statement about physical intuition than it is a poor man's choice: within the framework of a dynamical model with a distribution function, there is simply no other choice, for lack of adequate theories. One can show however that equilibrium models are fine for slowly evolving systems, if one translates the results to so called action space, but this is not my intention here.

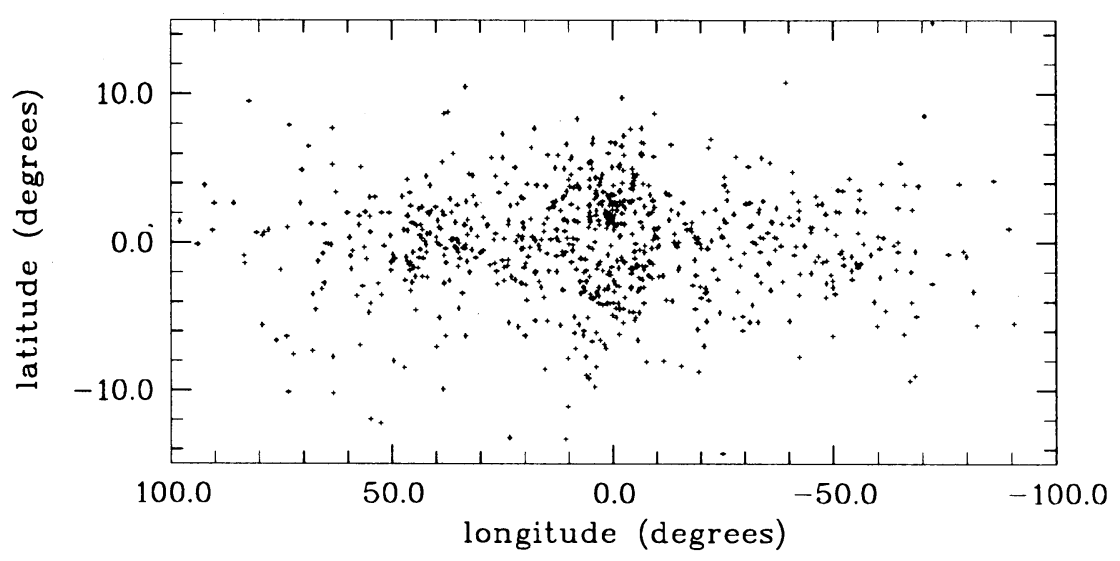

Fig. 2. The $(l, b)$ diagram of the tLH sample.

When studying the dynamics of a tracer population, it is obvious that selfconsistency is not required, i.e. the gravitational potential is not generated by the tracer population. This means that the gravitational potential can be decoupled from the original set of equations, and this potential therefore must be a given. The specification of the gravitational potential is the first important decision one has to make when building a dynamical equilibrium model. In particular, one has to decide on the prevailing geometry, i.e. whether the potential is spherical, axisymmetric or triaxial.

Figure 2 presents the $(l, b)$ distribution of the tLH sample. There is no obvious triaxiality in the data, which is also the case for the $(l, v)$ diagram in Figure 1. It's a very sad thing to note, especially since everybody now seems to detect more or less the same barlike structure; I suffice with referring to the index of the proceedings 
to illustrate this point. On the other hand, the absence of triaxiality in a tracer population does not mean that the potential isn't triaxial! Conversely, triaxiality in a tracer population does not imply triaxiality of the potential, but that is something you're not supposed to say... Well, anyway, let me say the unspeakable: I'll settle for axisymmetric potentials, and I'll forgive the very few who still have not quit reading for doing so now.

The IRAS satellite was severely confused in the GP, because of the high density of sources. In order to find $\mathrm{OH} / \mathrm{IR}$ stars there, one must resort to mapping type surveys. Lindqvist et.al. (1992a), hereafter L, searched for OH/IR in a limited region close to the GC, harvesting 134 stars. None of the surveys have been completely satisfactory in their velocity coverage, for technical and feasibility reasons. This means that (a few) high velocity stars can be expected to turn up when searching for them (van Langevelde 1992).
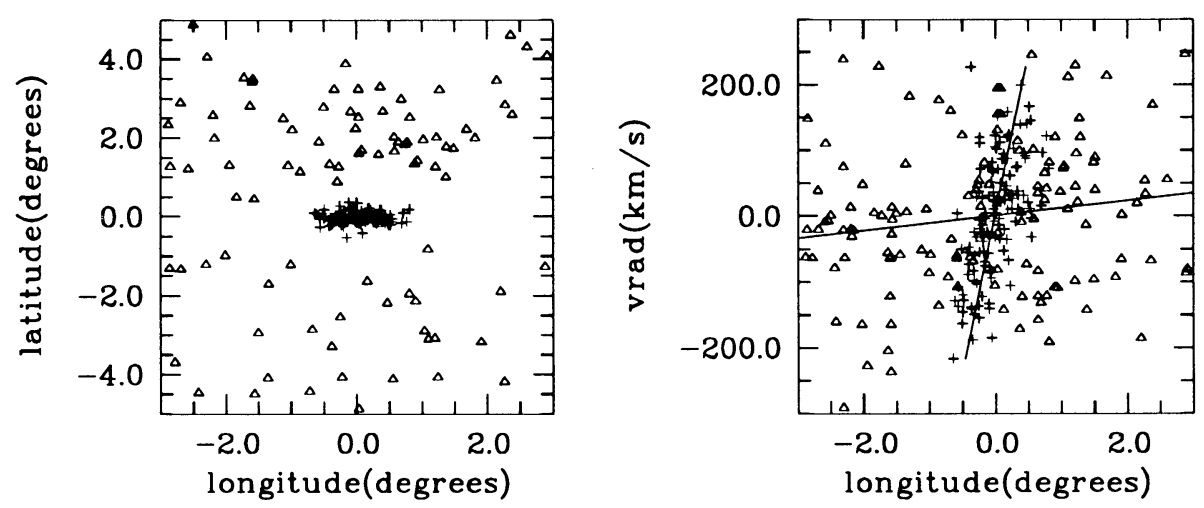

Fig. 3. (a) The L sample (crosses) and the truncated tLH sample (triangles) in $(\ell, b)$ on the sky. (b) The L sample (crosses) and the truncated tLH sample (triangles) in $\left(\ell, v_{r}\right)$ space.

I will not compare in any great detail the tLH and L samples. Some of that can be found in te Lintel Hekkert et.al. (1991b), and Lindqvist et.al. (1992b). Posters by Whitford and Winnberg, Lindquist \& Habing also address different aspects of this.

There is one observation I would like to make however, which is, I hope, strikingly clear in Figure 3. Figure 3a shows the $(\ell, b)$ diagrams for the $\mathrm{L}$ and the tLH samples, the latter being truncated to $\pm 3^{\circ}$ in longitude and $\pm 5^{\circ}$ in latitude. It is obvious that both samples are complementary, but certainly not enough so: the IRAS confusion zone is not covered. If the 2 samples are drawn from different galactic $\mathrm{OH} / \mathrm{IR}$ populations, we miss the data in the important transition region. Currently a consortium headed by Habing is working on surveys at the VLA and the AT to fill in a few gaps. Figure $3 \mathrm{~b}$ shows the $\left(\ell, v_{r}\right)$ plot for both samples (heliocentric velocities). The regression lines are the linear approximations to the "rotation curves". The $\mathrm{L}$ rotation curve (crosses) in this plot has a slope of about $500 \mathrm{~km} / \mathrm{s} /$ degree or about $3.7 \mathrm{~km} / \mathrm{s} / \mathrm{pc}$, using $R_{(:)}=7.5 \mathrm{kpc}$. On the ot? r hand, the slope of the tLH 
sample is $11 \mathrm{~km} / \mathrm{s} /$ degree or about $82 \mathrm{~km} / \mathrm{s} / \mathrm{kpc}$. Such a rotation curve reaches its presumed peak value of about $220 \mathrm{~km} / \mathrm{s}$ at about $2.5 \mathrm{kpc}$, which is very reasonable.

There are two quite different opinions here. The first one answers positively on the question whether the very different regression lines have anything to do with the sampling of different galactic populations. It is convincingly argued by Habing (this symposium) that the nature of the OH/IR's in both samples are different. If this also translates into the kinematics in such a way as it does here, than this connection would constitute one of the most dramatic links between abundance and kinematics that we know of. The other opinion would merely see the different regression lines as the result of a different dynamical environment. In particular, the $t L H$ regression line would be representative for the rotation curve as we know it, while the $\mathrm{L}$ regression line would indicate a (fairly modest) mass concentration in the very center. Values are given in Lindqvist et.al. (1992b). This second opinion implies that we would do well not to combine both samples, unless there is a mass concentration provided in the potential.

There is a lot to say about the nature of the OH/IR stars, and whether we can differentiate the class into different galactic populations (Habing, this symposium). For example, the conjecture by Baud et al. (1981) and Olnon et al. (1981) that the velocity of the expanding circumstellar shell may indicate an age, in the (statistical) sense that larger expansion velocities are associated with younger stars, has been a working hypothesis ever since it was first proposed. It looks like this simple picture may need amendment, and therefore I will in the sequel indicate this by quoting "old" and "young".
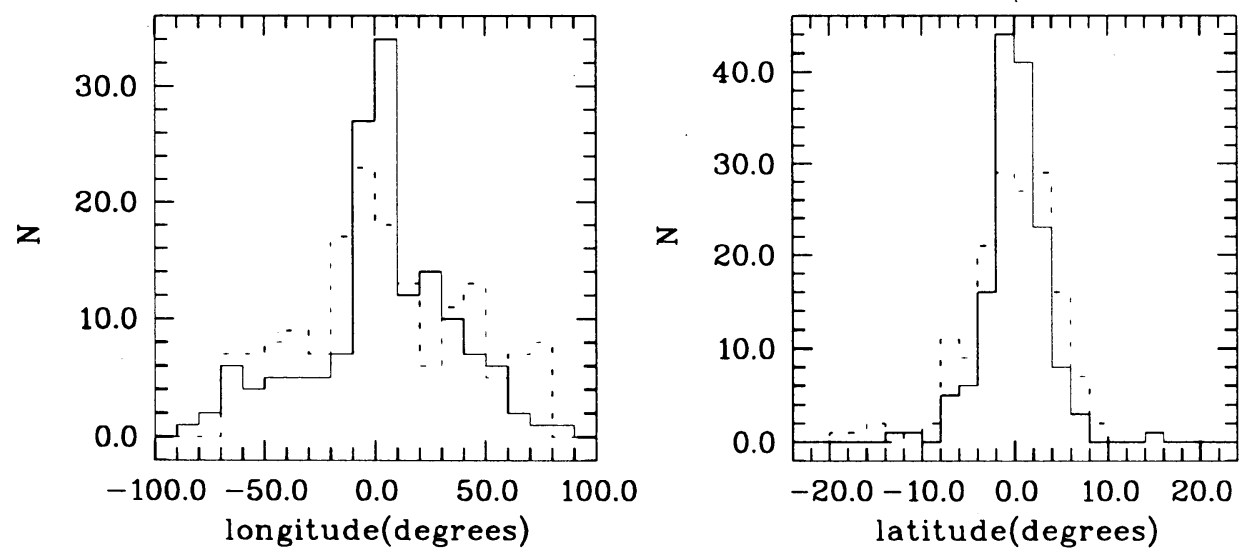

Fig. 4. The histograms of "old" stars (shells expanding at a rate between 10 and $13 \mathrm{~km} / \mathrm{s}$ ) in the tLH sample (dashes) and the "young" stars (solid lines, shells with expansion velocities between 16 and $20 \mathrm{~km} / \mathrm{s}$ ), as a function of galactic longitude and latitude

In any case, trom the analysis by Lindqvist et.al. (1992b) it would seem that indeed the projected velocity dispersion of the "younger" stars is smaller than the dispersion for the "older" stars, as one could expect if stars are born on primarily circular orbits. In Figure 4 we see histograms as a function of longitude and latitude for a selection of the tLH sample, divided into two groups defined by the "oldest" 
and "youngest" stars, each containing about 150 stars. If the aforementioned effect is real, it points in any case into the right direction: the "younger" population is more confined to the disk, and somewhat more bulgy, i.e. centrally concentrated. Whether you believe it or not may depend on whether you are an astronomer or a statistician.

Finally, since the available positions and radial velocities do not give much information on the $z$-component of the velocity for stars in the GP, it is natural to try two integral models first (based on the specific binding energy $E$ and the $z$ component of the angular momentum, which are both integrals of the motion in an axisymmetric potential). Such models have the property that $\sigma_{r}=\sigma_{z}$. Only when the projected velocity dispersion turns out to be much too small (since for twointegral models $\sigma_{r}$ is determined by the thinness of the disk), will we be able to rule out two-integral models. In view of Kent's (1992) success in modelling the Bulge with 2-integral hydrodynamical models, it is instructive to see whether this result holds when 2-integral models are considered that go all the way to the distribution function.

\section{The model: method and results}

So far we have made modelling choices based on arguments that could be deduced from the data alone. Now it is time to consider the options that are rather a matter of preference.

We need an explicit potential function. For this we used either a Stäckel fit to the Bahcall-Soneira potential (Dejonghe \& de Zeeuw 1988) or a simple Stäckel halo-disk potential of the type discussed by Batsleer \& Dejonghe (this symposium). The Stäckel fit is not needed at this point, but will come in handy when 3 integral models are made, which must be done eventually. It is important to note however that the adopted potentials all more or less reproduce the Burton \& Gordon (1978) peak at .5 kpc. Recent analysis (Burton \& Liszt 1993), allowing for non-circular motions, puts into question this peak, reminding us how little we know for sure about the potential of the Bulge.

We need a distance to the GC. We adopt $7.5 \mathrm{kpc}$, for no particular reason.

From the sample we can construct a body of data that can be very inhomogeneous, including star counts, mean velocities, velocity dispersions and line profiles..., on a few selected patches in the sky. The left panels in Figure 5 are smoothed renditions of the data, based on running averages (counts, mean velocities and velocity dispersions) over the 15 closest data points, which for this figure were selected among the "youngest" stars. The white (or light gray) areas correspond to the regions where there are no data because of completeness problems (IRAS confusion) or simply because of paucity of data points. The left panels were used to create the input data for the modelling.

No general theorem exists that would enable us to decide on the uniqueness of the distribution function for such data, and few analytic procedures are known to construct it. These procedures are applicable only in special cases (e.g. homogeneous data on the mass density, see a contribution by Hunter (this symposium)). Therefore, it might be useful to consider a method that is more or less independent 

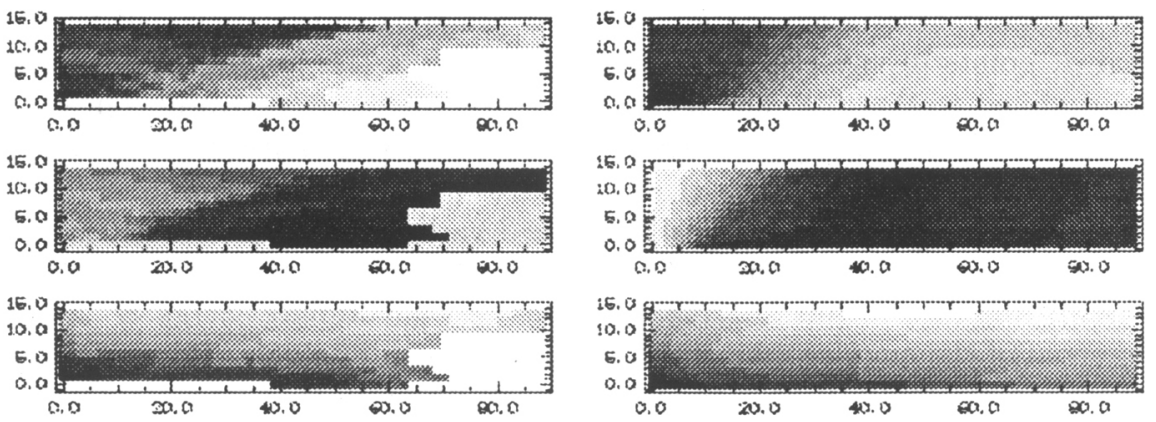

Fig. 5. A qualitative comparison of data (left panels) and model (right panels) on the sky, produced for the sample with highest circumstellar expansion velocities. The bottom panels represent the logarithm of the counts per square degree; the gray scales range from $-1.5 /$ sq.deg. to $1 . /$ sq.deg. The middle panels are the (projected) mean velocity fields, with values from $0 \mathrm{~km} / \mathrm{s}$ to $220 \mathrm{~km} / \mathrm{s}$. The top panels represent the (projected) velocity dispersions, gray scales range from $10 \mathrm{~km} / \mathrm{s}$ to $115 \mathrm{~km} / \mathrm{s}$.

of the diversity in the data.

In almost all cases the relation of this distribution function with the observable quantities takes on the form of an average of the distribution function:

$$
\mu_{o b}\left(\mathbf{x}_{\ell}, \mathbf{v}_{\ell}\right)=\int_{\tau_{\ell}} \mu(\mathbf{x}, \mathbf{v}) F\left(E, I_{2}, I_{3}\right) d \mathbf{x} d \mathbf{v}
$$

with $\left(\mathbf{x}_{\ell}, \mathbf{v}_{\ell}\right)$ a label for a region in the space of observables (e.g. a region on the sky, a region in $\left(\ell, v_{r}\right)$ space. . $), \tau_{\ell}$ the region in phase space that contributes to the mean, and $\mu_{o b}$ the observable (counts/sqare degree, mean velocity,...) The operator is linear, which means that we can construct models by superposition.

One way to proceed is with Quadratic Programming (Dejonghe 1989). In this method it is assumed that the distribution function can be written as a linear combination of (preferably analytically simple) components, with coefficients $c_{i}$. A $\chi^{2}$-type function (quadratic in the $c_{i}$ ) is then mimimized, subject to the constraint that the distribution function must be positive everywhere (linear constraints in the $c_{i}$ ). We also need to pick a series of basis functions to construct the linear combination, and these were Fricke components, which are basically powers in energy and angular momentum, and disk components, of the type discussed by Batsleer \& Dejonghe (this symposium). For additional details, see also te Lintel Hekkert et.al. (1991b).

The right panels in Figure 5 show the model fits to the data (in the left panels). The gray scales give at least a qualitative idea properties of the fit. The areas where there are no data are now filled in (let's call them predictions, or extrapolations if you insist). In figure 6 a more quantitative comparison is presented. Clearly, one should not expect the fit to reproduce all details in the data, which may not be all real anyway. Problem areas may be the projected velocity dispersion for the "old" stars, which is somewhat too low, and the mean velocity which rises too fast for 

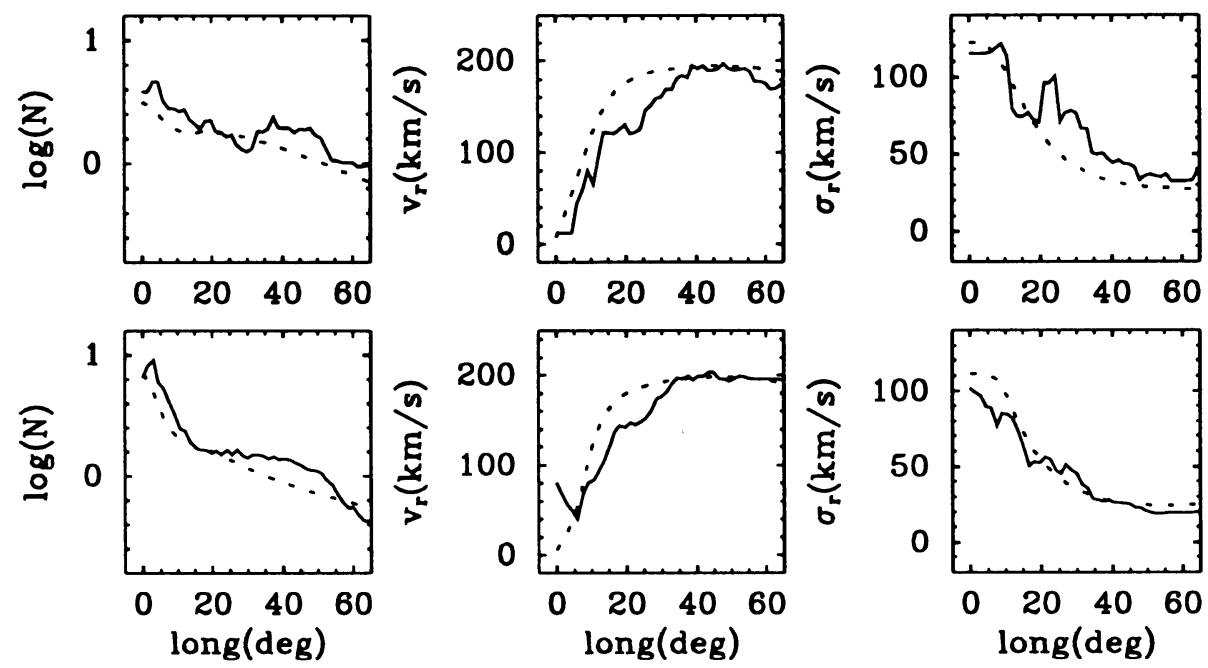

Fig. 6. The data at $b=2^{\circ}$ (solid lines) compared with a 2-integral model (dashes). Left panels: logarithm of projected star counts per square degree, middle panels: projected mean velocity, right panels: projected velocity dispersions. Top panels: the "old" stars, bottom panels: the "young" stars.
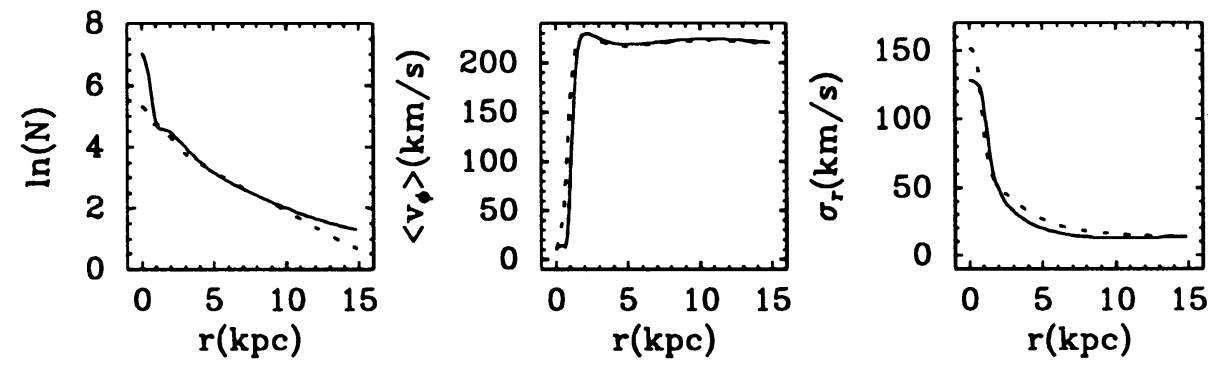

Fig. 7. The logarithm of the spatial density in the plane, the mean rotation and the radial velocity dispersion for the "young" (solid) and "old" (dashed) stars

both samples. This may indicate the need for a third integral.

In Figure 7, the spatial number density, mean rotation and radial velocity dispersion are plotted for both groups of stars. The "old" stars show a nice exponential disk with scale factor $3.5 \mathrm{kpc}$. This is no artifact of the components used in QP, since none of them showed exponential behaviour but were rather polynomial. The mean rotation follows very closely the rotation curve. This is a consequence of the 2-integral models, which, in order to produce fairly flat disks, must have small velocity dispersion. The "old" stars have overall a somewhat higher velocity dispersion, which clearly shows a bulge component.

Finally, Figures 8 and 9 show the distribution function in turning point space and in integral space (the color versions are much nicer!). All well-known compo- 


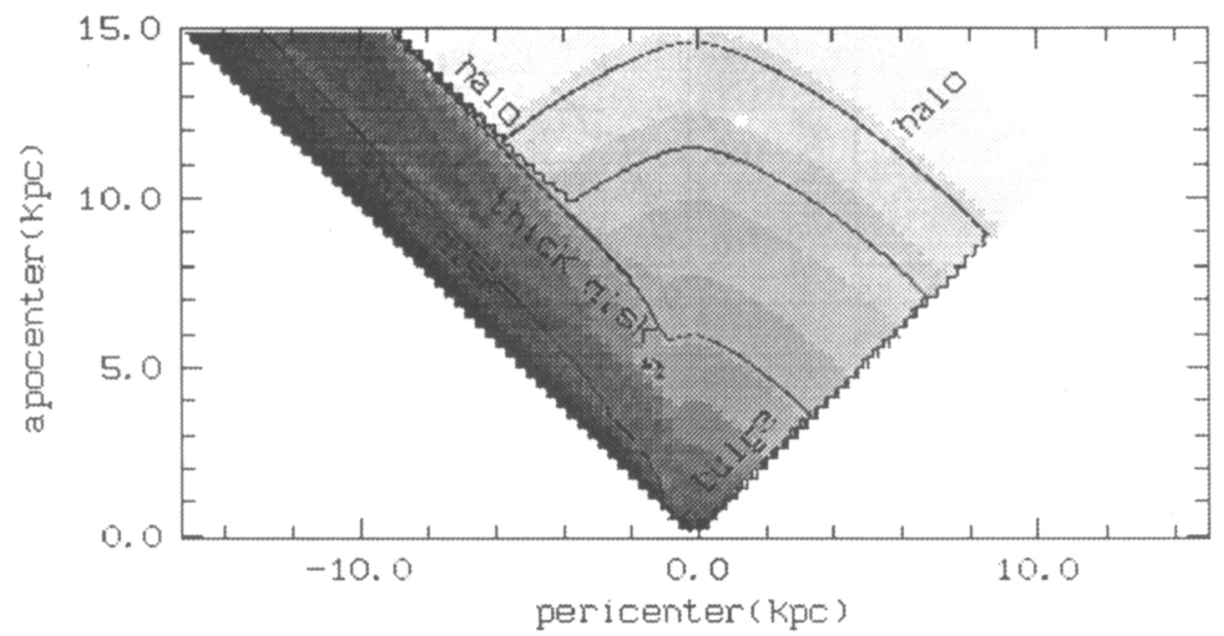

Fig. 8. The distribution function in turning point space. Every point inside the wedge-like region corresponds to an orbit with apocenter and pericenter as implied by the axes. Negative pericenter simply means negative angular momentum. Contours are chosen to delineate the different components. The dynamic range is of the order $10^{12}$.

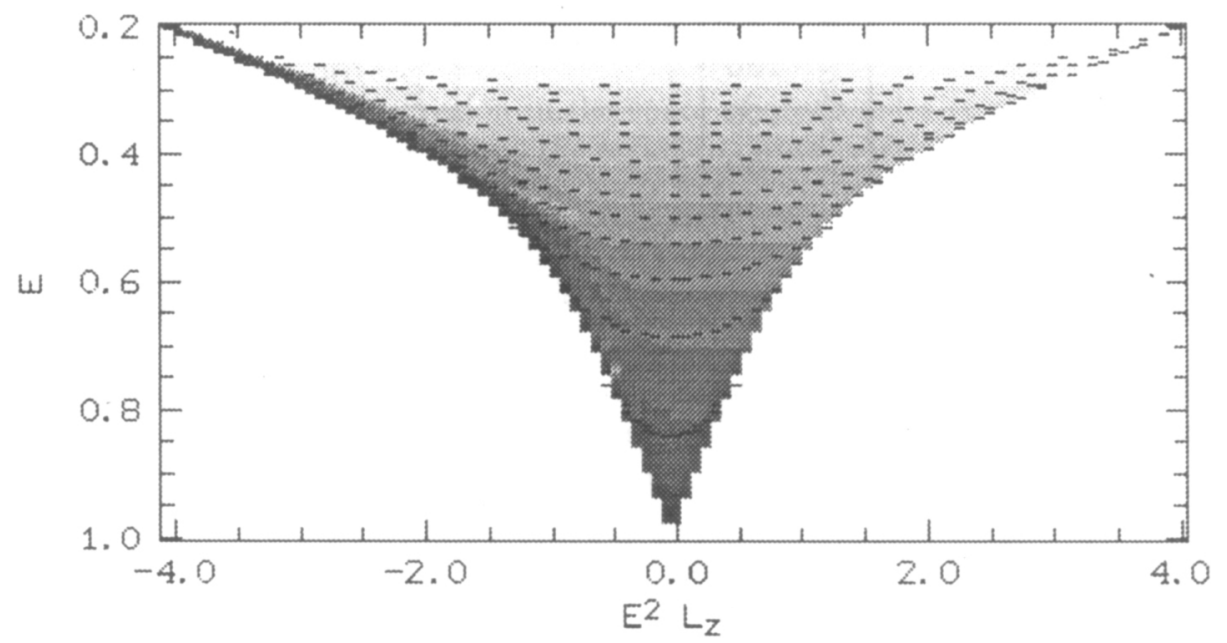

Fig. 9. The distribution function in integral space. Every point inside the wedge-like region corresponds to an orbit with integrals as implied by the axes. The boundaries of the region are the loci of the circular orbits. The distribution function is the same as in Figure 8 . The dotted contours are loci of constant apocenter, given from $1 \mathrm{kpc}$ (bottom) to $15 \mathrm{kpc}$ (top). 
nents are present, and are indicated in the turning point plot. The dynamic range is very large: the highest value is about $10^{7} \mathrm{stars} / \mathrm{kpc}^{3} /(\mathrm{km} / \mathrm{s})^{3}$ in the thin disk, but, obviously, such values are very uncertain.

It is possible to make predictions on the basis of these models. For example, since we know the distribution function, all observable kinematical quantities can be calculated. As an example, Figure 10 depicts the line profiles (i.e. the $(l, v)$ diagram) in the GP, drawn from the above distribution function for the "younger" $\mathrm{OH} / \mathrm{IR}$ 's, superposed on the sample. This calculation is, in a sense, a prediction, since there has been no fitting on the $(l, v)$ diagrams directly. As is obvious, the fit is very reasonable.

\section{Conclusions}

The OH/IR stars are excellent probes of our dynamical Galaxy. They are fairly old, strong infrared emittors that are reasonably representative of a relaxed population. As such, they can be modelled with equilibrium dynamical models. Their strong infrared emission makes them shine right through the dusty GP, a property which is needed for a sufficient spatial coverage.

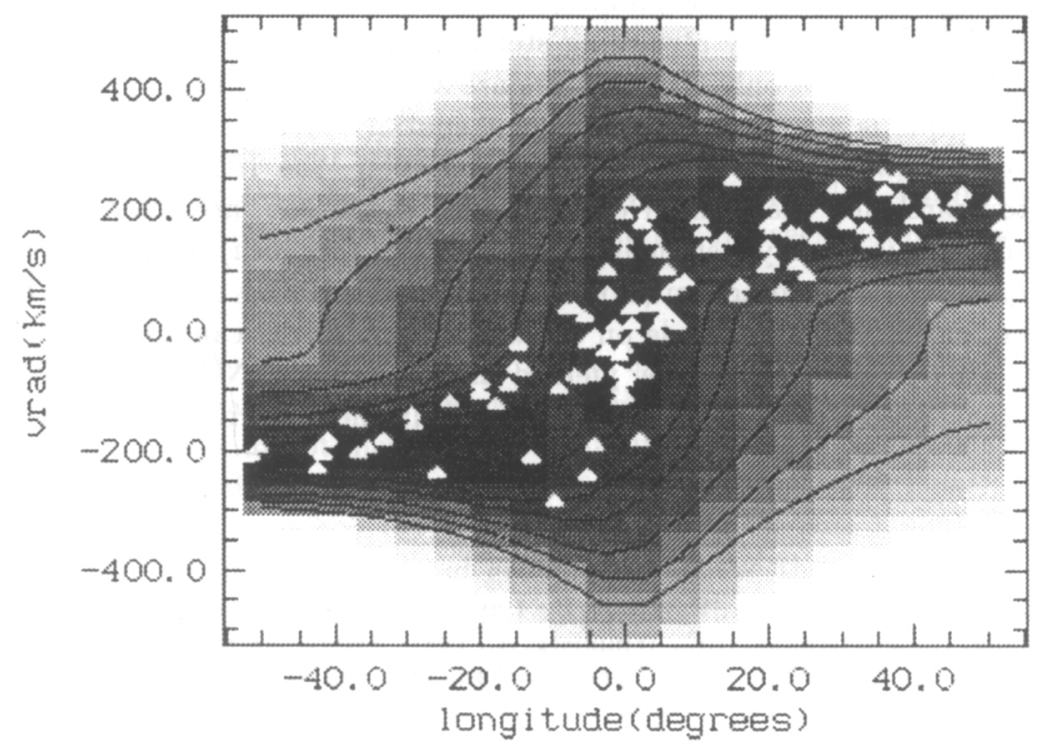

Fig. 10. The $(l, v)$ diagram for the above distribution function, together with the sample for which it was calculated.

Differentiation of the sample towards a definition of galactic populations is possible, but no firm conclusions can be drawn, as yet.

Only numerical experience at this point can give us an idea to what degree we can have confidence in the computed distribution function. It is obvious that the more the data cover phase space, the more the distribution function will be constrained. Also, the more restrictive we are in the functional form of $F$ (function 
of one, two, or three integrals), the less realistic our results may be, but the less indeterminacy we will encounter when trying to determine a distribution function. And this is, by the way, is an important reason to start with 2-integral models!

Two integral models are reasonable fits, but it is already clear that eventually three integral models will be needed. This is indicated by the fit of the model in the transition region between Bulge and Disk, where the flatness of the Disk sets fairly strong constraints on the kinematics in a 2-integral approach. This is somewhat in contrast with Kent's (1992) results, possibly because the positivity of the distribution function is taken into account here. As soon as proper motions become available (see e.g. Spaenhauer, Jones \& Whitford 1992, Minniti, this symposium) on large areas in the sky, global models such as these will almost certainly need 3-integral dynamics.

The adopted potential is still very unsure. Especially for the Bulge regions, the potentials used in this analysis are probably inadequate. It would be worthwhile to reconsider the models with Bulge potentials of the kind used by Kent (1992) and Burton \& Liszt (1993). In a more remote future, simple theoretical models for orbits in rotating barlike potentials may be needed, if the bar in the Bulge turns out to be dynamically significant for the stellar populations.

This contribution is only the beginning of what I consider to be long and laborious but potentially extremely rewarding stellar dynamical modelling of Galactic. populations. Moreover, not only $\mathrm{OH} / \mathrm{IR}$ stars are amenable to this kind of analysis. Whenever a sample is available with sufficient spatial coverage, it is presumably worthwhile to try analyses of this kind. In particular, the IRAS PSC has also been used to search for Planetary Nebulae, since these, too, are strong infrared emittors, and occupy a fairly well defined place in the $f_{\nu}(12 \mu \mathrm{m}) / f_{\nu}(25 \mu \mathrm{m})$ versus $f_{\nu}(25 \mu \mathrm{m}) / f_{\nu}(60 \mu \mathrm{m})$ color diagram (Pottash et.al. 1988, Ratag et.al. 1990). Radio interferometry can be used to decide on the true nature of the candidates (Zijlstra et.al. 1989). This method up to now yielded about 50 new PNs within $15^{\circ}$ from the galactic center, on a total of about 400 in roughly the same region (Acker et.al. 1991).

\section{References}

Acker, A., Köppen, J., Stenholm, B., Raytchev, B., 1991, Astron. Astrophys. Suppl., 89, 237

Baud, B., Habing, H.J., Matthews, H.E., Wimberg, A., 1981, Astron. Astrophys., 95, 171.

Bimmey, J. \& Tremaine, S., 1987, Galactic Dynamics, Princeton University Press

Burton, W.B. \& Gordon, M.A., 1978, Astron. Astrophys., 63, 7

Burton, W.B. \& Liszt, H.S., 1993, Astron. Astrophys., submitted

Dejonghe, H., 1989, Astrophys. J., 343, 113

Dejonghe, H. \& de Zeeuw, P.T., 1988, Astrophys. J., 329, 720

Eder, J., Lewis, B.M., Terzian, Y., 1988, Astrophys. J. Suppl., 66, 183

Goodman, J. \& Schwarzschild, M., 1981, Astrophys. J., 245, 1087

Kent, S, 1992, Astrophys. J., 387, 181

Lindqvist, M., Winmberg, A., Habing, H.J., Matthews, H.E., 1992a, Astron. Astrophys. Suppl, 92 , 43

Lindqvist, M., Habing, H.J., Wimmberg, A., 1992b, Astron. Astrophys., 259, 118

Olnon, F.M., Walterbos, R.A.M., Habing, H.J., Matthews, H.R., Winnberg, A., Brzezinska, H., Baud, B., 1981, Astrophys. J., 245, L103.

Pottash, S.R., Bignell, C., Olling, R., Zijlstra, A.A., 1988, Astron. Astrophys., 205, 248

Ratag, M.A., Pottash, S.R., Zijlstra, A.A., Menzies, J., 1990, Astron. Astrophys., 233, 181 
Sivagnanam, P., Braz, M.A., Le Squeren, A.M., Tran Minh, F., 1989, Astron. Astrophys., 211, 341.

Spaenhauer, A., Jones, B.F. \& Whitford, A.E., 1992, Astron. J., 103, 297

te Lintel Hekkert, 1990, Ph. D. thesis, Leiden

te Lintel Hekkert, P., Caswell, J.L., Habing, H.J., Norris, R.P., Haynes, R.F., 1991a, Astron. Astrophys. Suppl., 90, 327

te Lintel Hekkert, P., Dejonghe, H., Habing, H.J., 1991b, Proc. of Astron. Soc. of Austr., 9, 20

van Langevelde, H.J., Brown, A.G.A., Lindqvist, M., Habing, H.J., de Zeeuw, P.T., 1992, Astron. Astrophys. Lett., submitted

Zijlstra, A.A., Pottash, S.R., Bignell, C., 1989, Astron. Astrophys. Suppl., 79, 329

\section{JOINT DISCUSSION (HABING AND DEJONGHE)}

de Zeeuw: How. well does your distribution function for the Bulge, the two integral one, compare to simple forms that have been proposed in the literature, for example by Rowley, for other bulges?

Dejonghe: The model that comes out for the Bulge is fairly isotropic. To that degree, since Rowley's models can also be isotropic, there is likely to be agreement. I haven't at this stage checked this though, but we could probably find at least one set of parameters for his models that would produce a qualitative fit. But producing a quantitative fit, that's another matter!

Rich: If you separate your sample out just by expansion velocity, that is including the Winnberg sample, do you see the Winnberg kinematic disk falling out naturally or is there some other means of your distinction between young and old $\mathrm{OH} / \mathrm{IR}$ stars?

Dejonghe: The Wimnberg sample is much less extended. So I'm not sure that this kind of comparison could be easily made. But answering your question: the Winnberg disk would not merely fall out naturally, simply on the basis of selection on expansion velocity.

Rich: te Lintel had a group I and group II. I believe that group II is thought to be younger. How does that group II compare to the Winnberg stars, is it a lower expansion?

Habing: They are overlapping, so there is no clear distinction on that basis.

Sellwood: You claimed there was no evidence for triaxiality in your sample. But you threw out the one piece of evidence that is there, and that is the rapid rotation of the Winnberg sample. Surely you are looking down a bar and that is exactly what the Winnberg rapid rotation is telling us. 
Dejonghe: This model does not use the Winnberg sample. Judging from the te Lintel sample, there is no obvious need for a triaxial bulge and it very well may be that the rapid rotation of the Winnberg sample could be due to high mass, but, then again, it might be a bar. For the big picture, out to $15 \mathrm{kpc}$ (in the te Lintel sample), I see no need for triaxiality.

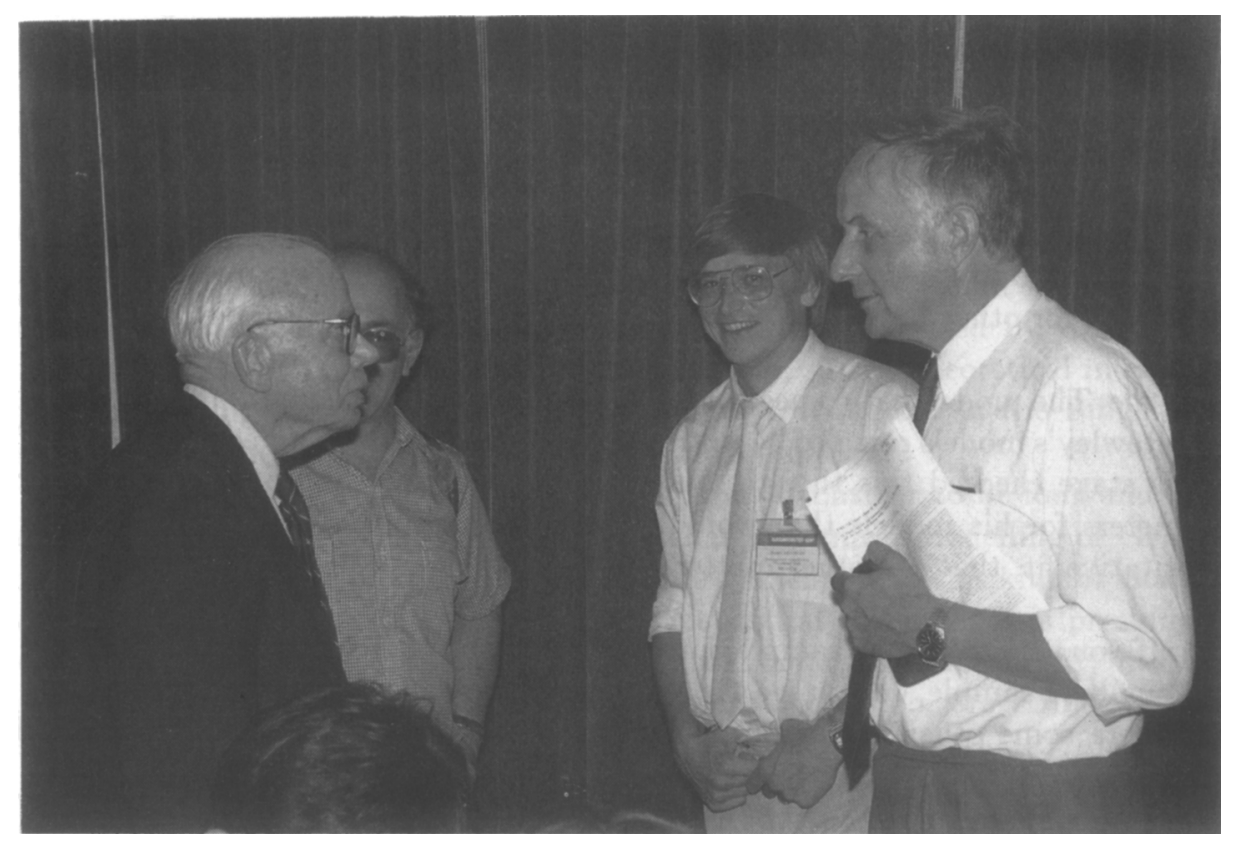

A. Whitford, together with K. Freeman, H. Dejonghe and H. Habing 